\title{
TERAPIA FOTODINÁMICA EN ESTRÍAS ANGIOIDES
}

\section{PHOTODYNAMIC THERAPY IN ANGIOID STREAKS}

\author{
ELÍAS-DE-TEJADA M M ${ }^{1}$, CALVO-GONZÁLEZ $\mathrm{C}^{1}$, RECHE-FRUTOS J ${ }^{1}$, DONATE-LÓPEZ J ${ }^{1}$, \\ GARCÍA-SÁNCHEZ J²
}

\section{RESUMEN}

Objetivo: Evaluar la eficacia y seguridad de la Terapia Fotodinámica (TFD) en la neovascularización coroidea (NVC) asociada a estrías angioides (EA).

Métodos: Se realiza un estudio retrospectivo de ocho pacientes (once ojos) diagnosticados de EA, que presentan neovascularización subfoveal, yuxtafoveal o extrafoveal, tratada al menos una vez con TFD. Se obtiene la mejor agudeza visual corregida (MAVC), retinografía así como angiografía con fluoresceína (AFG), antes y después del tratamiento para valorar su eficacia. El seguimiento medio fue de 13,2 meses.

Resultados: Diez ojos presentaban membranas subfoveales, un ojo yuxtafoveal y otro extrafoveal. Tras la TFD, la AV se mantuvo igual en dos pacientes, aumentó en un paciente y disminuyó en ocho pacientes. En cuanto a la respuesta angiográfica, nueve ojos presentaron respuesta fibrótica tras una sesión de TFD, mientras que en dos ojos persistió exudación. Hubo tres casos de recidiva de la NVC. Conclusiones: La TFD en la NVC asociada a EA no parece alterar el curso de esta enfermedad, sólo enlentece su progresión.

Palabras clave: Estrías angioides, terapia fotodinámica, neovascularización coroidea.

\begin{abstract}
Objetive: To evaluate the efficacy and safety of photodynamic therapy in choroidal neovascularization associated with angioid streaks.

Methods: We performed a retrospective study of eleven eyes (eight patients) with subfoveal, juxtafoveal or extrafoveal neovascularization, which received at least one session of photodynamic therapy. Treatment efficacy was evaluated by visual acuity (VA) testing, color photography and fluorescein angiography. The mean follow-up was 13.2 months. Results: Ten eyes presented subfoveal neovascularization, one eye presented juxtafoveal and another eye extrafoveal neovascularization. VA was unchanged in two patients, increased in one patient, and decreased in eight patients following photodynamic therapy. Nine eyes presented conversion to a fibrotic disciform lesion, whereas leakage persisted in two eyes after one session of photodynamic therapy. Recurrence of choroidal neovascularization appeared in three eyes.

Conclusions: Photodynamic therapy in choroidal neovascularization related to angioid streaks does not appear to alter the course of this disease, but it does slow its progression (Arch Soc Esp Oftalmol 2007; 82: 741-746).
\end{abstract}

Key words: Angioid streaks, photodynamic therapy, choroidal neovascularization.

\footnotetext{
Recibido: 2/10/06. Aceptado: 17/9/07.

Departamento de Retina del Hospital Clínico San Carlos. Madrid. España.

1 Licenciado en Medicina.

2 Doctor en Medicina.

Correspondencia:

M. Elías de Tejada Casanova

Servicio de Oftalmología. Retina. Hospital Clínico San Carlos

C/. Profesor Martín Lagos, s/n

28040 Madrid

España

E-mail: macaelias@yahoo.es
} 


\section{INTRODUCCIÓN}

Evaluar la eficacia y seguridad de la terapia fotodinámica (TFD) en el tratamiento de la neovascularización coroidea asociada a estrías angioides (EA).

Las EA son roturas en la membrana de Bruch que aparecen en la funduscopia como bandas anaranjadas o grisáceas alrededor del disco óptico, por ser en este punto dónde la musculatura ocular extrínseca ejerce mayor tracción, y desde allí tienen una extensión radial (fig. 1). Existe una serie de patologías que se asocian al desarrollo de EA como son el pseudoxantoma elástico, la enfermedad de Paget, la elastosis senil cutánea y la fibrodisplasia hiperplástica o síndrome de Ehlers-Danlos.

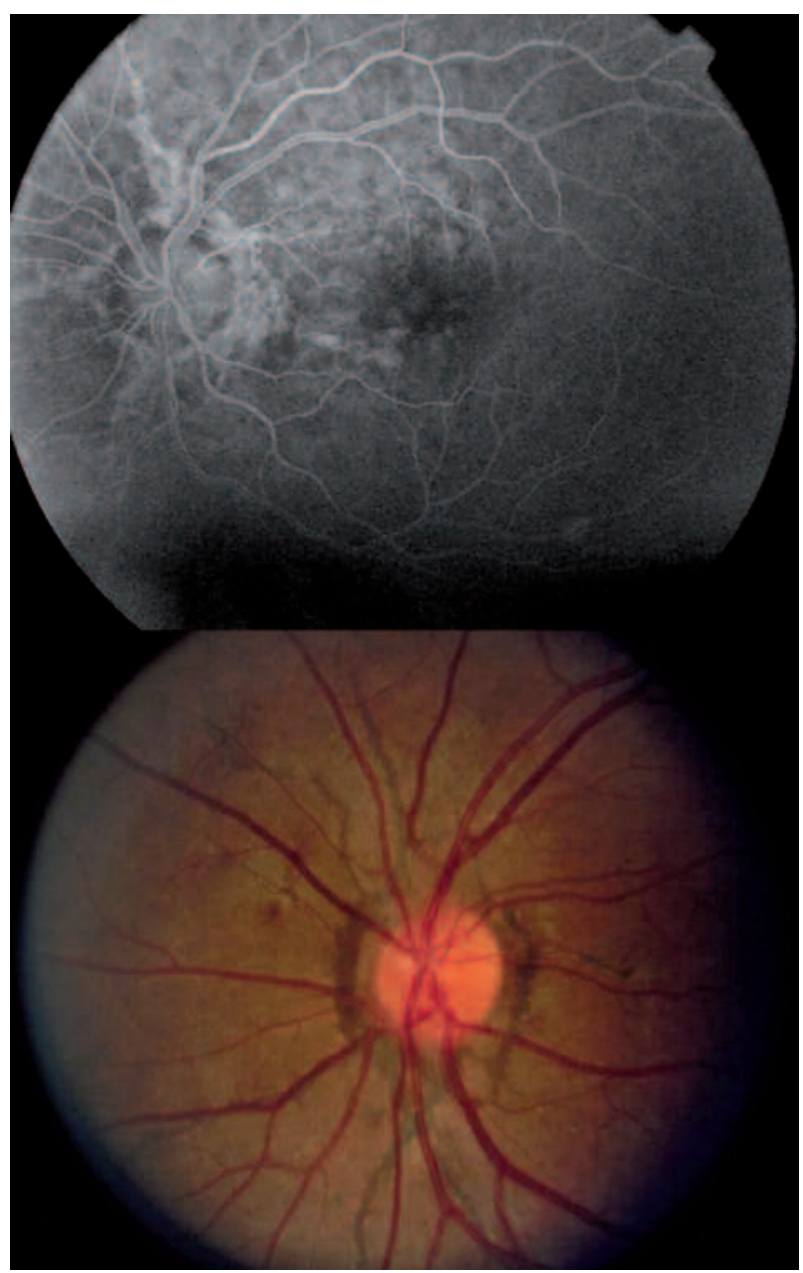

Fig. 1: Retinografía y Angiografía fluoresceínica depaciente con estrías angioides. Se aprecia en la angiografía hiperfluorescencia precoz y tardía a nivel de las estrías angioides.
La importancia de las EA reside en el desarrollo tardío de neovascularización coroidea (NVC), originando mayoritariamente membranas subfoveales clásicas. La patogénesis del desarrollo de estas membranas es semejante a la de la NVC existente en la retinopatía miópica: a través de las roturas en la membrana de Bruch penetran los vasos de la coroides hacia el epitelio pigmentario de la retina (EPR) y el espacio subretiniano.

Cuando se desarrolla la NVC, los pacientes empiezan a tener clínica, con disminución de agudeza visual (AV) y metamorfopsias. En la campimetría aparece un escotoma central y en las pruebas de visión cromática se altera el eje azul-amarillo (1).

En el diagnóstico de las EA juega un papel importante la Angiografía con fluoresceína (AFG), que nos ayuda asimismo a detectar la aparición de NVC.

Existen diferentes posibilidades terapéuticas para la NVC asociada a estrías angioides, tales como la fotocoagulación con láser árgon, la cirugía macular y la TFD.

La fotocoagulación con láser árgon de los neovasos se emplea en membranas yuxtafoveales y extrafoveales. Asocia un alto número de recidivas así como el desarrollo de escotomas centrales de gran tamaño, secundarios al daño producido en el tejido sano, al ser éste un láser no selectivo (2).

La cirugía consiste en la extracción de la membrana neovascular y traslocación macular, asociada a un gran porcentaje de complicaciones (3).

La terapia fotodinámica (TFD) con verteporfino se ha empleado en la NVC secundaria a DMAE, Miopía patológica y otras causas.

El objetivo del presente estudio es valorar la eficacia de la TFD en la NVC secundaria a EA.

\section{SUJETOS, MATERIAL Y MÉTODOS}

Se presenta un estudio retrospectivo de ocho pacientes (once ojos) con membranas neovasculares coroideas activas angiográficamente asociadas a EA.

El diagnóstico de la NVC se realizó en todos los casos mediante una AFG, clasificando las membranas, en función de su patrón angiográfico en clásicas u ocultas y según su localización en extrafoveales, yuxtafoveales o subfoveales. En nuestro estudio se diagnosticaron nueve membranas clásicas y dos ocultas, de las que nueve estaban localizadas subfoveolares, y las dos restantes extra y yuxtafoveolares respectivamente (fig. 2). 
La mejor agudeza visual corregida (MAVC) antes de iniciar el tratamiento oscilaba entre 20/200 y 20/40, con una media de 20/80.

La TFD se aplicó según el protocolo convencional consistente en Verteporfín (Visudyne ${ }^{\circledR}$; Novartis Ophthalmic, Hettlinger Suiza), diluído en solución salina balanceada y dextrosa al 5\% para conseguir una dosis del fármaco de $6 \mathrm{mg} / \mathrm{m}^{2}$ de superficie corporal y un volumen total de infusión de $30 \mathrm{ml}$. La solución se administró vía intravenosa a una velocidad de $3 \mathrm{ml} / \mathrm{min}$ durante alrededor de 10 minutos. El láser se aplicó a los 15 minutos del inicio de la infusión. El tamaño del spot del láser se determinó en función del eje mayor de la lesión, y añadiendo $1.000 \mathrm{~mm}$ a ese valor. El tiempo para administrar $50 \mathrm{~J} / \mathrm{cm}^{2} \mathrm{de}$ láser fue de 83 segundos.
Los pacientes fueron revisados cada 12 semanas, y en cada revisión se determinó la AV según el ETDRS (Early Treatment Diabetic Retinopathy Study), y se les realizó una retinografía y una AFG para estudiar la actividad de la NVC. En caso de exudación de la membrana se dio otra sesión de TFD. El número de sesiones de TFD osciló en nuestra serie entre una y tres, con una media de 1,33 sesiones.

El tiempo medio de seguimiento fue de 13,2 meses (rango entre 3 y 24 meses).

\section{RESULTADOS (tabla I)}

Se estudiaron 8 pacientes, 3 hombres y 5 mujeres cuya edad media fue de 56,87 años (rango, 43-78).

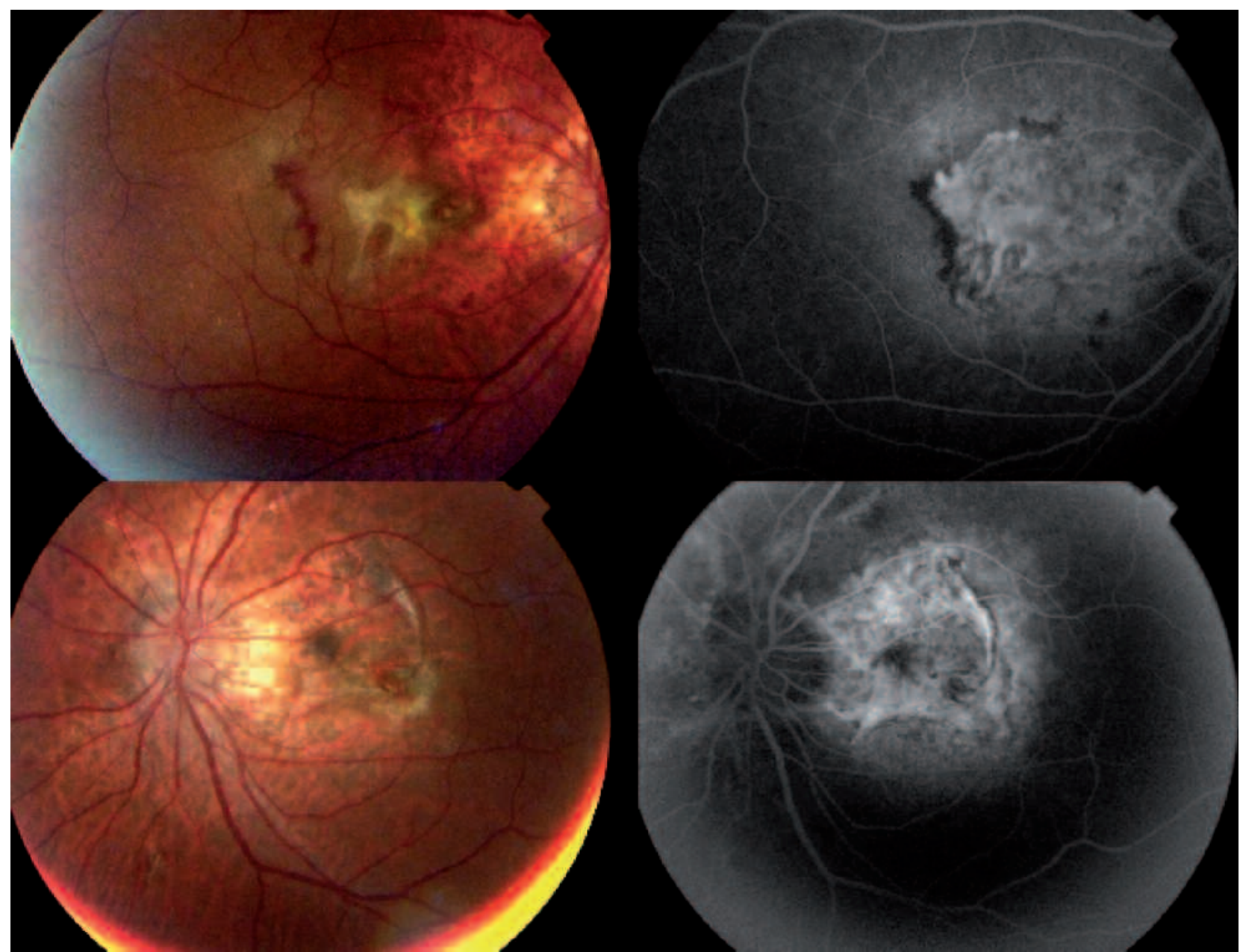

Fig. 2: Caso 1. Retinografía y angiografía antes de TFD, se observa neovascularización coroidea subfoveal en ambos ojos. 
Tabla I. Características clínicas de la población de nuestro estudio

\begin{tabular}{lccccccccc}
\hline $\begin{array}{l}\text { Caso } \\
\text { número }\end{array}$ & Edad & Sexo & Ojo & PXE & $\begin{array}{c}\text { N. }^{\circ} \text { sesiones } \\
\text { TFD }\end{array}$ & $\begin{array}{c}\text { Seguimiento } \\
\text { (meses) }\end{array}$ & AV inicial & AV final & AFG final \\
\hline 1 & 67 & V & OD & + & 2 & 24 & $20 / 200$ & CD & Fibrótica \\
1 & 67 & V & OI & + & 1 & 24 & $20 / 100$ & $20 / 200$ & Fibrótica \\
2 & 50 & M & OD & - & 1 & 6 & $20 / 60$ & $20 / 100$ & Mínima exudación \\
2 & 50 & M & OI & - & 1 & 6 & $20 / 40$ & $20 / 60$ & Fibrótica \\
3 & 59 & M & OI & - & 1 & 19 & $20 / 200$ & CD & Fibrótica \\
3 & 59 & M & OD & - & 1 & 19 & $20 / 125$ & $20 / 125$ & Fibrótica \\
4 & 45 & M & OI & + & 2 & 12 & $20 / 60$ & CD & Cicatricial \\
5 & 78 & V & OD & - & 2 & 17 & $20 / 100$ & CD & Cicatricial \\
6 & 55 & M & OI & + & 1 & 12 & $20 / 60$ & $20 / 80$ & Mínima exudación \\
7 & 58 & V & OI & + & 1 & 3 & $20 / 100$ & $20 / 60$ & Fibrótica \\
8 & 43 & M & OI & - & 1 & 3 & $20 / 200$ & $20 / 200$ & Fibrótica \\
\hline \hline
\end{tabular}

PXE: Pseudoxantoma elástico; V: Varón; M: Mujer; OD: Ojo derecho; OI: Ojo izquierdo; CD: Cuenta dedos; AV en Snellen.

Su MAVC media pretratamiento fue de 20/80 (rango 20/200-20/40).

Tras el tratamiento, la MAVC se mantuvo en 2 casos (18\%), disminuyó en 8 casos (73\%) y aumentó en 1 caso (9\%). La AV final media fue 20/200 (rango cuenta dedos-20/60).

La exudación de la NVC tras TFD disminuyó o desapareció en todos los casos tras una sesión de TFD, observándose una respuesta fibrótica (fig. 3) en 9 ojos (82\%), 3 de los cuales $(27 \%)$ evidenciaron reactivación de la lesión (fig. 4) en un ojo, la recidiva apareció al año del tratamiento, y en los otros 2 a los 6 meses de la sesión de TFD. En los 3 casos, tras una segunda sesión de TFD desapareció la exudación de la NVC.

Sólo en 2 ojos (18\%) persistió exudación, siendo necesaria otra sesión de TFD.

\section{DISCUSIÓN}

Aún siendo poco común, el impacto de la NVC asociada a estrías angioides es grande, ya que causa escotomas centrales, y aunque los pacientes, en su mayoría jóvenes, mantienen una buena visión periférica queda dificultada su vida laboral. Sin tratamiento, estas lesiones progresan rápidamente hacia la ceguera.

Se ha utilizado la fotocoagulación con láser árgon para NVC yuxtafoveal y extrafoveal asociada a EA, observándose remisión de la exudación, pero escotomas grandes y recidivas en un alto porcentaje de los casos. En cualquier caso, esta modalidad de tratamiento no era recomendable en NVC subfoveal (2).
El uso de la TFD para el tratamiento de membranas subfoveales en miopía y degeneración macular asociada a edad (DMAE) con resultados satisfactorios, especialmente en membranas clásicas, abrió un nuevo horizonte en el tratamiento de esta patología.

La TFD consiste en la inyección de un agente fotosensibilizante, el verteporfino, seguido de su activación con una luz de láser no térmico, en su pico de absorción, a $690 \mathrm{~nm}$. Al ser excitado el agente sensibilizante, genera radicales de oxígeno y otros productos que causan daño en la membrana endotelial de los neovasos, produciendo trombosis de éstos.

En pacientes con NVC secundaria a DMAE, se vio que la exudación recurría a las 12 semanas de una sesión de TFD, pero el grupo TAP (Treatment of Age-related macular degeneration with Photodynamic Therapy) publicó que la incidencia de recidiva de la NVC era menor en las sucesivas sesiones de TFD (4).

En nuestro trabajo se encontró una pérdida de AV del $58 \%$ y una persistencia de la exudación determinada angiográficamente en un $17 \%$. Se han observado resultados similares en diversos estudios (5-7), mientras que otros autores presentan resultados más optimistas sin disminución de AV postratamiento y falta de actividad de la NVC tras una sesión de TFD (8).

En conclusión, la TFD en la NVC asociada a estrías angioides no mejora la AV final de los pacientes ni modifica el tamaño de la NVC. No obstante, parece enlentecer la progresión de la enfermedad al disminuir la exudación de las membranas.

Se han descrito recidivas de la neovascularización coroidea tras TFD, aunque en menor porcentaje que con la fotocoagulación con láser argón. 


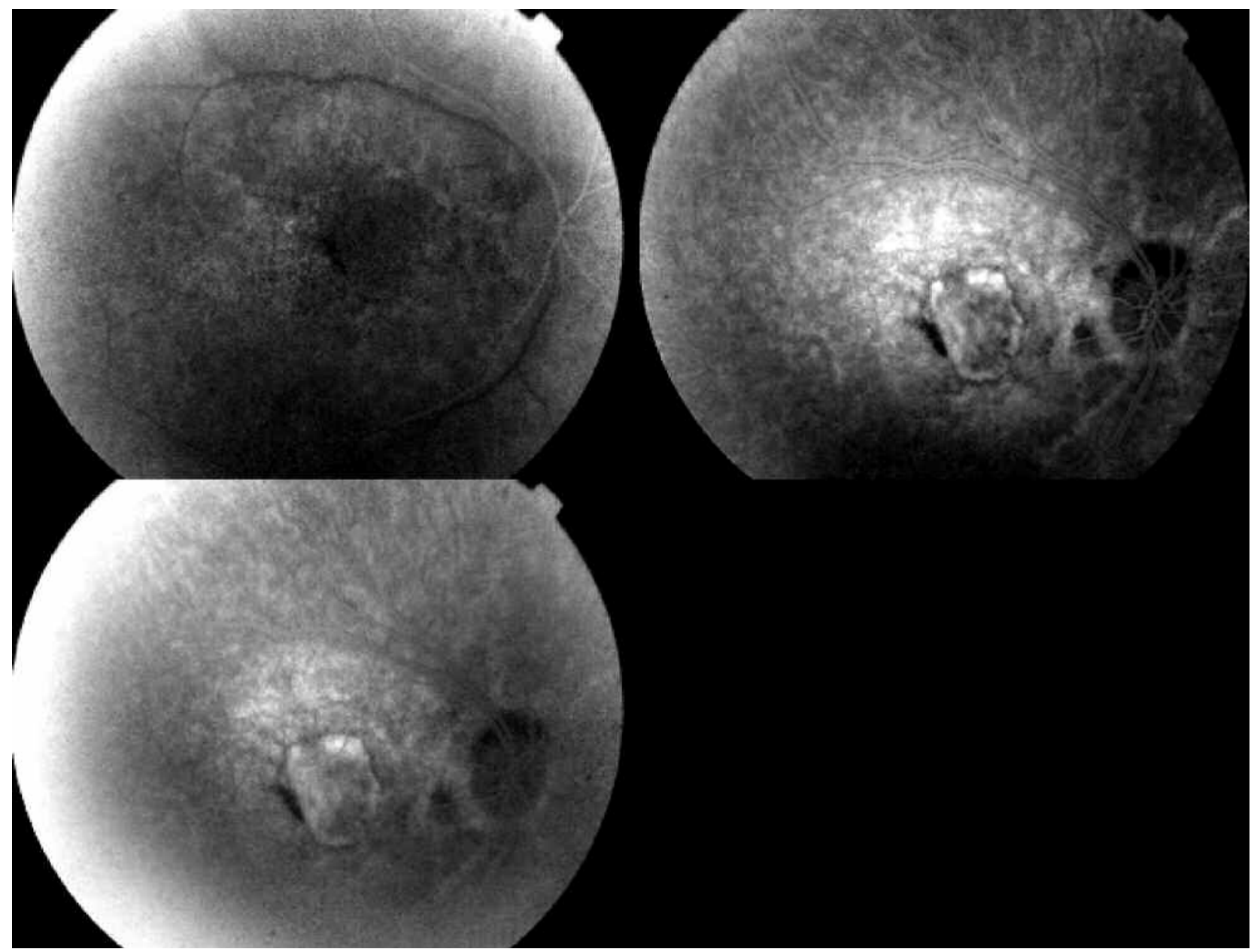

Fig. 3: Caso 1. Angiografía post TFD: respuesta fibrótica.

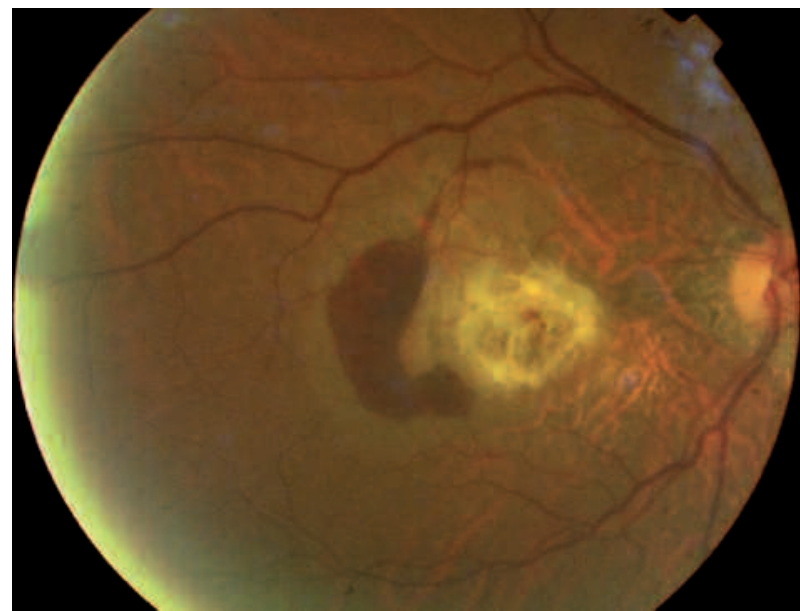

Fig. 4: Caso 5. Retinografía mostrando recidiva de la neovascularización coroidea. Se visualiza sangre y exudación al lado de cicatriz disciforme.

\section{BIBLIOGRAFIA}

1. Deutman AF, Hoyng CB. Macular dystrophies. In: Ryan SJ. Retina. St Louis: Mosby; 2001; 1242-1244.

2. Costa RA, Calucci D, Cardillo JA, Farah ME. Selective occlusion of subfoveal neovascularization in angioid streaks by using a new technique of ingrowth site treatment. Ophthalmology 2003; 110: 1192-1203.

3. Roth DB, Estafanous M, Lewis H. Macular translocation for subfoveal choroidal neovascularization in angioid streaks. Am J Ophthalmol 2001; 131: 390-392.

4. Verteporfin In Photodynamic Therapy Study Group. Verteporfin therapy of subfoveal choroidal neovascularization in agerelated macular degeneration: two-year results of a randomized clinical trial including lesions with occult with no classic choroidal neovascularization-verteporfin in photodynamic therapy report 2. Am J Ophthalmol 2001; 131: 541-560.

5. Arias L, Pujol O, Rubio M, Caminal J. Long-term results of Photodynamic Therapy for the treatment of choroidal neovascularization secundary to angioid streaks. Graefes Arch Clin Exp Ophthalmol 2006; 244: 753-757. 
6. Shaikh S, Ruby AJ, William GA. Photodynamic therapy using verteporfin for choroidal neovascularization in angioid streaks. Am J Ophthalmol 2003; 135: 1-6.

7. Browning AC, Chung AK, Ghanchi F, Harding SP, Musa$\operatorname{dig} M$, Talks SJ, et al. Verteporfin photodynamic therapy of choroidal neovascularization in angioid streaks: one- year results of a prospective cases series. Ophthalmology 2005; 112: 1227-1231.

8. Karacorlu M, Karacorlu S, Ozdemir H, Mat C. Photodynamic therapy with verteporfin for choroidal neovascularization in patients with angioid streaks. Am J Ophthalmol 2002; 134: 360-366. 